\title{
Swift multi-wavelength observations of the bright flaring burst GRB 051117A
}

\author{
M. R. Goad ${ }^{1}$, K. L. Page ${ }^{1}$, O. Godet ${ }^{1}$, A. Beardmore ${ }^{1}$, J. P. Osborne ${ }^{1}$, P. T. O’Brien ${ }^{1}$, R. Starling ${ }^{1}$, S. Holland ${ }^{2}$, \\ D. Band ${ }^{2}$, A. Falcone ${ }^{3}$, N. Gehrels ${ }^{2}$, D. N. Burrows ${ }^{3}$, J. A. Nousek ${ }^{3}$, P. W. A. Roming ${ }^{3}$, A. Moretti ${ }^{4}$, and M. Perri ${ }^{5}$ \\ 1 Department of Physics and Astronomy, University of Leicester, LE1 7RH, UK \\ e-mail: mrg@star.le.ac.uk \\ 2 NASA Goddard Space Flight Center, Greenbelt, MD 20771, USA \\ 3 Pennsylvania State University, University Park, PA 16802, USA \\ 4 INAF-Osservatorio Astronomica di Brera, via Bianchi 46, 23807 Merate (LC), Italy \\ 5 ASI Science Data Center, Via Galileo Galilei, 00044 Frascati, Italy
}

Received 5 December 2006 / Accepted 15 March 2007

\section{ABSTRACT}

\begin{abstract}
We report on the temporal and spectral characteristics of the early X-ray emission from the Gamma Ray Burst 051117A as observed by Swift. The superb quality of the early X-ray light-curve and spectra of this source, one of the brightest seen by the X-ray Telescope at such early times, allows an unprecedented look at the spectral and temporal evolution of the prompt and early afterglow emission for this GRB and allows us to place stringent limits on the detection of lines. GRB 051117A displays a highly complex light-curve, with an apparent initial slow decline of slope $\alpha=0.77 \pm 0.07\left(f(t) \propto t^{-\alpha}\right)$ dominated by numerous superposed flares of varying amplitude and duration. Between orbits 2 and 3, the X-ray light-curve drops abruptly, highlighting the dominance of flaring activity at early times, and indicating that the central engine for this burst remains active for several kiloseconds after the initial explosion. The late time slope ( $t>10^{4} \mathrm{~s}$ ) also decays relatively slowly with a powerlaw index of $\alpha=0.66$, breaking to a steeper slope of $1.1,170 \mathrm{ks}$ after the BAT trigger. The X-ray light-curve at early times is characteristic of a noise process, consisting of random shots superposed on an underlying powerlaw decay, with individual shots well-modelled by a fast-rise and exponential decay spanning a broad range in rise-times and decay rates. A temporal spectral analysis of the early light-curve shows that the photon index and source intensity are highly correlated with the spectrum being significantly harder when brighter, consistent with the movement of the peak of the Band function to lower energies following individual flares. The high quality spectrum obtained from the first orbit of WT mode data, enables us to place a $3 \sigma$ upper limit on the strength of any emission line features of $E W<15 \mathrm{eV}$, assuming a narrow emission-line of $100 \mathrm{eV}$ at the peak of the effective area.
\end{abstract}

Key words. gamma rays: bursts - gamma rays: observations

\section{Introduction}

The Swift Gamma-Ray Burst Explorer (Gehrels et al. 2004), now approaching the end of its second year of operations, is routinely observing the prompt gamma-ray and early afterglow emission of Gamma-Ray Bursts (GRBs) in the astrophysically important minutes to hours timescale after the onset of the burst. The greater sensitivity over previous gamma-ray missions of the Burst Alert Telescope (hereafter BAT, Barthelmy 2004; Barthelmy et al. 2005a) together with Swift's unique autonomous pointing and rapid slew capability enables observations of the burst position in the narrow-field instruments, the X-Ray Telescope (XRT, Burrows 2004; Burrows et al. 2005a) and UltraViolet-Optical Telescope (UVOT, Roming et al. 2005) on timescales of less than $100 \mathrm{~s}$, opening up to scrutiny a largely unexplored region of parameter space.

Amongst Swift's many outstanding successes during the first two years of operations, are the following notable highlights: the first localisation of the afterglow emission from a short GRB (e.g. Gehrels et al. 2005), the detection of a very high redshift burst (e.g. Cusumano et al. 2006), an unexpected rapid decline phase in the prompt X-ray light-curves (Tagliaferri et al. 2005; Goad et al. 2006b), observations of frequent flaring activity in the early X-ray light-curves of approximately half of all bursts
(Burrows et al. 2005b; King et al. 2005; Falcone et al. 2006), and the intriguing discovery of a burst which would have been classified as short by the Burst and Transient Source Experiment (BATSE) on the Compton Gamma-Ray Observatory (CGRO), but had a long soft tail in the BAT which lasted over $100 \mathrm{~s}$ (e.g. Barthelmy et al. 2005a), to name but a few. Indeed the many exciting new discoveries by Swift have posed many challenges to existing theoretical models of the prompt and early afterglow emission of GRBs.

In the standard fireball model (see e.g. Zhang \& Mészáros 2004 for a thorough review), the core-collapse of a massive star produces a relativistically expanding blast wave which decelerates as it impacts on the surrounding interstellar medium. The GRB spectrum is dominated by non-thermal emission (either synchrotron or inverse Compton) from shock accelerated relativistic electrons, which subsequently cool as the fireball expands, causing the spectrum to shift toward lower energies.

Large amplitude, short timescale fluctuations in both the prompt $\gamma$-ray and early X-ray light-curves as observed by Swift, support the suggestion that the GRB prompt emission arises from internal processes rather than from external shocks (i.e. before the blastwave has been decelerated by the ambient medium). Possible scenarios include production via internal shocks (Rees \& Mészáros 1994; Kobayashi et al. 1997), 
dissipation in strong magnetic fields (Drenkhahn \& Spruit 2002; Kumar et al. 2007) or Comptonization of the photospheric emission (Rees \& Mészáros 2005).

Indeed, the early rapid declines in the X-ray light-curves, and the smooth connection between the early X-ray light-curves and the tail of prompt $\gamma$-ray emission (Barthelmy et al. 2005a), suggests that the early X-ray emission is simply an extension of the prompt $\gamma$-ray emission seen in the initial explosion (Nousek et al. 2006). The steep decay slopes $\left(f(t) \propto t^{-3}\right.$ or steeper) associated with the prompt X-ray emission (e.g. Tagliaferri et al. 2005; Goad et al. 2006b) are also consistent with internal processes. If the central engine activity ceases abruptly, an external observer will continue to see emission from increasingly large angles $\left(\theta>\Gamma^{-1}\right)$ with respect to their line-of-sight, the so-called "curvature effect" (e.g. Kumar \& Panaitescu 2000; Dermer 2004; Fan \& Wei 2005). This steep phase is typically followed by a shallower decay phase which is spectrally harder (e.g. Goad et al. 2006b; Nousek et al. 2006; Willingale et al. 2007) and is thought to be associated with late time energy injection (refreshed shocks) which suggests the central engine activity may last for far longer than had previously been thought. At later times the light-curve may steepen to follow the canonical afterglow decay value $(\alpha \approx 1.2-1.4)$ observed in the decay of optical afterglow light-curves. Interestingly, jet-breaks accompanying the late time decay of the X-ray afterglow are for the most part not detected by Swift (e.g. Willingale et al. 2007).

At least one Swift GRB has an associated Supernovae (GRB 060218/SN2006aj, Campana et al. 2006a). Given the observed apparent association between some GRBs and SN (see e.g. Woosley \& Bloom 2006, and references therein), there has long been the expectation that emission-lines would be detected in the early X-ray and optical afterglow spectra of GRBs. Indeed, there have been numerous claims in the literature for the detection of X-ray emission-lines in the X-ray spectra of GRB afterglows across a number of X-ray platforms including BeppoSax, Chandra and XMM (e.g. Piro et al. 1999; Piro et al. 2000; Yoshida et al. 2001; Reeves et al. 2002; Butler et al. 2003; Watson et al. 2003), though in the majority of cases the detection significance is modest (a few $\sigma$ ) at best. Line species identified include $\mathrm{FeK} \alpha$, with an energy consistent with the redshift of the host galaxies, and/or lines associated with blue-shifted lighter elements of $\mathrm{S}, \mathrm{Si}, \mathrm{Ar}, \mathrm{Mg}$ and $\mathrm{Ca}$. Two possible scenarios have been proposed for the origin of the X-ray emissionlines. In the first, post-burst energy injection is reprocessed into line emission by material lying very close to the GRB progenitor $\left(R \sim 10^{13} \mathrm{~cm}\right)$ (Rees \& Mészáros 2000). In this model the lifetime for the line-emission is governed by the duration of the post-burst injection phase. Alternatively, the line emission may be formed by reprocessing of both the prompt and early afterglow emission at relatively large distances $\left(R \sim 10^{16} \mathrm{~cm}\right.$, e.g. Lazzati et al. 2002; Reeves et al. 2002; Kumar \& Narayan 2003). In this scenario, the lifetime of the emission is determined by the size of the reprocessing region (see e.g. Gao \& Wei 2005 for an in-depth discussion of this model). Futhermore, the line emission is expected to be detected within the first few hours of the initial explosion. To date despite early ( $<$ few minutes) X-ray and optical observations of more than 150 GRBs by Swift, there is as yet no conclusive evidence for line emission in any of the afterglow spectra (see e.g. Butler et al. 2007; Hurkett et al. 2007).

Here we report on Swift observations of the bright flaring burst GRB 051117A, one of the brightest bursts observed with XRT. The paper is organised as follows. In Sect. 2 we describe the data obtained by Swift. In Sect. 3 we present a detailed temporal and spectral analysis of the BAT and early XRT data for this burst. In Sect. 4 we present observations of GRB 051117A in other bands. In Sect. 5 we place the observations in the context of theoretical models of the GRB and afterglow emission. Our conclusions are presented in Sect. 6.

\section{Observations}

\subsection{GRB 051117A}

The Swift BAT triggered and located on board GRB 051117A (trigger $=164268$ ) at 10:51:20 UT Nov. 17th 2005 (Band et al. 2005). The spacecraft autonomously slewed to the burst location and began observations with XRT at 10:53:07 UT. The XRT found a very bright, fading, uncatalogued source at RA 15h13m33.8s, Dec +30d52m13.3s (J2000), with a positional uncertainty of $3.4 \operatorname{arcsec}(90 \%$ containment, Goad et al. 2005). This position which includes the latest XRT boresight correction (Moretti et al. 2006), lies 41 arcsec from the BAT onboard position and 3.8 arcsec from the refined UVOT position (RA 15h13m34.1s, Dec +30d52m12.7s (J2000), Holland 2005).

\subsubsection{BAT spectrum and light-curve of GRB $051117 \mathrm{~A}$}

The BAT light-curve of GRB 051117A is characterised by a long FRED-like (Fast Rise Exponential Decay) peak at $T_{0}-15 \mathrm{~s}$ lasting out to at least $T_{0}+190 \mathrm{~s}$, with a hint of smaller emission peaks at $T_{0}+225 \mathrm{~s}$ and $T_{0}+350 \mathrm{~s}$ (each of approximately $30 \mathrm{~s}$ in duration). A simple powerlaw fit to the BAT spectrum, covering the time interval $T_{0}-29 \mathrm{~s}$ to $T_{0}+157 \mathrm{~s}$, has a best-fit photon in$\operatorname{dex} \Gamma=1.83 \pm 0.07$, with a fluence of $4.6 \pm 0.16 \times 10^{-6} \mathrm{erg} \mathrm{cm}^{-2}$ (all values in the $15-150 \mathrm{keV}$ band). The peak flux in a $1 \mathrm{~s}$ wide window starting at $T_{0}+2.47 \mathrm{~s}$ is $0.93 \pm 0.17 \mathrm{ph} \mathrm{cm}^{-2} \mathrm{~s}^{-1}$ (quoted errors are $90 \%$ confidence on 1 interesting parameter). Relative to other Swift detected GRBs, GRB 051117A is rather unremarkable, with a BAT fluence placing it at the mean of the brightness distribution, while its spectral characteristics place it at the soft end of the burst population. In fact based on Lamb's definition (Lamb et al. 2005), GRB 051117A qualifies as an X-ray Rich Burst.

\subsubsection{XRT observations of GRB 051117A}

The XRT began observing GRB 051117A $107 \mathrm{~s}$ after the BAT trigger. The on-board software located a very bright uncatalogued X-ray source in a single $0.1 \mathrm{~s}$ Image Mode (IM) frame, with an initial flux estimate of $1.0 \times 10^{-8} \mathrm{erg} \mathrm{cm}^{-2} \mathrm{~s}^{-1}$ in the $0.2-10 \mathrm{keV}$ band (this assumes an unabsorbed powerlaw model with photon index $\Gamma=2.0$ ).

GRB 051117A is amongst the brightest (in terms of observed counts) early X-ray light-curves yet observed by Swift. Indeed, the source was so bright in XRT $\left(>200 \mathrm{ct} \mathrm{s}^{-1}\right.$ in the IM frame) and the initial rate of decline so slow ( $\alpha=0.77$, where $f(t) \propto t^{-\alpha}$ ), that the source remained in Windowed Timing (WT) mode for the whole of the first orbit, providing an unprecedented $1770 \mathrm{~s}$ of WT mode data in the first 2 orbits. XRT switched into Photon Counting (PC) mode $170 \mathrm{~s}$ into the 2 nd orbit of observations (5187 s after the BAT trigger). We note that even in orbit 2, the PC mode data are piled-up, which is highly unusual for data taken at such a late time. For later orbits, XRT remained in PC mode and followed the source until it faded into the background, with a total on source exposure time of $\approx 430 \mathrm{ks}$ (see Table 1 for details of the observations).

The WT and PC mode event lists were processed using the standard XRT data reduction software xrtpipeline 
Table 1. Swift XRT observing log.

\begin{tabular}{cccr}
\hline \hline Obsid & $\begin{array}{c}T_{\text {start }} \\
\text { MET }(\mathrm{s})\end{array}$ & $\begin{array}{c}T_{\text {stop }} \\
\text { MET }(\mathrm{s})\end{array}$ & $\begin{array}{c}T_{\exp } \\
(\mathrm{s})\end{array}$ \\
\hline 00164268000 & 153917592.991 & 153924985.900 & $4092.9^{\dagger}$ \\
00164268001 & 153934003.854 & 153974578.652 & 14893.0 \\
00164268002 & 153974610.738 & 154051198.756 & 30341.0 \\
00020022002 & 154055821.405 & 154305538.688 & 57527.0 \\
00020022004 & 154310791.615 & 154392358.971 & 21640.0 \\
00020023002 & 154397100.274 & 154479238.316 & 22902.0 \\
00020023003 & 154483724.965 & 154739637.492 & 64452.0 \\
00020023004 & 154743975.915 & 154912439.176 & 20419.0 \\
00020023005 & 155004275.072 & 155086497.067 & 18321.0 \\
00020023006 & 155091100.675 & 155173374.540 & 16275.0 \\
00020023007 & 155177778.735 & 155259236.189 & 21247.0 \\
00020023008 & 155264657.803 & 155519998.411 & 70525.0 \\
00020023009 & 155520621.431 & 155589836.745 & 7767.5 \\
00020023010 & 155693247.237 & 155775479.364 & 19183.0 \\
00020023011 & 155780139.059 & 155862711.964 & 19581.0 \\
00020023012 & 155866953.038 & 155971765.193 & 20783.0 \\
\hline
\end{tabular}

$\dagger$ Includes both WT and PC mode data.

version 0.9.4, within FTOOLS v6.0.3, screening for hot and flickering pixels, bad columns, and selecting event grades $0-12$ for PC mode data, 0-2 for WT mode data. Table 1 lists the name, start and stop times (in mission elapsed time, MET) and total on source exposure time of each observing segment. Observations of GRB 051117A were continued until Dec. 11th 2005 , yielding 24 days of coverage. Due to the close proximity of GRB 051117A to the Sun direction, there were no extensive ground-based follow up observations of this source in the immediate days to weeks after the burst. Fortunately, the location of GRB 051117A was in a favourable sky position for XRT observations $^{1}$, and the CCD temperature remained cool for the duration of our observations.

In Fig. 1 we show the central few arcminutes of a deep ( 430 ks) XRT observation of the field of GRB 051117A, formed from the summed cleaned PC event lists from orbit 3 onwards. There are 99 sources in the field detected at $>5$ sigma. Of particular note is the bright stellar source at RA $15 \mathrm{~h} 13 \mathrm{~m} 36.4$, Dec +30d52m57.9s (J2000) which lies only 54 arcsec from the XRT position of the GRB. Due to the close proximity of this source to the GRB we used a smaller than typical source extraction region for PC mode data, a circular aperture of outer radius 15 pixels ( $\equiv 35.4$ arcsecs), compared to the default 20 pixel radius region used in the standard pipeline analysis. Similarly, for the WT mode data we use a 30 pixel wide box centred on the source (cf. the default 40 pixel wide box). Due to the large number of background sources, the background region had to be chosen with care. For the PC mode data we choose a circular background region of 55 pixel radius located at RA $15 \mathrm{~h} 13 \mathrm{~m} 58.3 \mathrm{~s}$ Dec $+30 \mathrm{~d} 50 \mathrm{~m} 22.3 \mathrm{~s}$ (J2000, uncorrected for boresight), and which appears to be free from contamination by background sources (see Fig. 1). For the WT mode data we choose a background region with the same size as the source

\footnotetext{
${ }^{1}$ Failure of the XRT Thermo Electric Cooler (TEC) during the calibration and verification phase of the mission has introduced a change to the mission operations, with an additional workload on the observation planners, with the requirement that GRB observations must maintain the XRT temperature below -47 degrees Celsius. This essentially means choosing space-craft roll angles which keep the radiator pointed away from the Earth and other non-favourable directions. For higher temperatures hot and flickering pixels increase substantially and can result in frequent and undesirable mode switching.
}

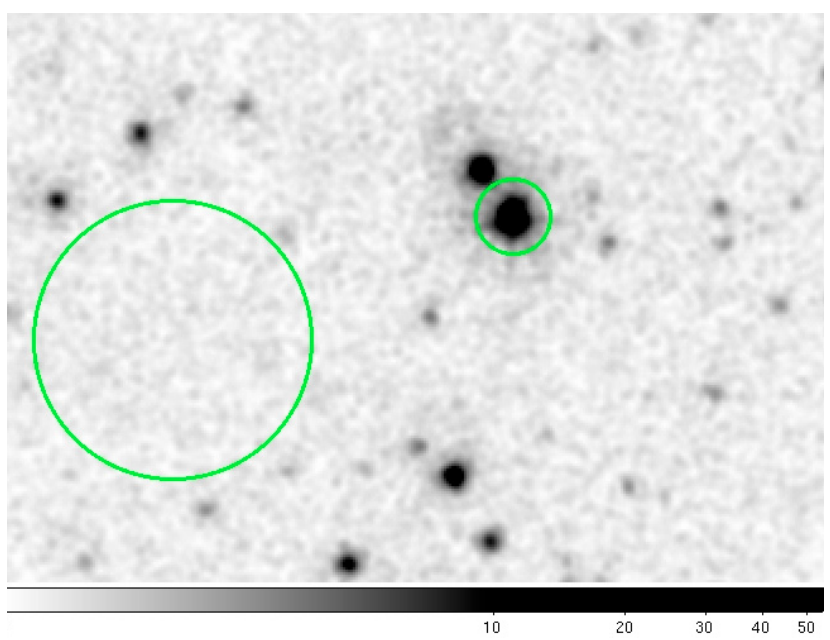

Fig. 1. A deep, smoothed, XRT image formed from summing the cleaned event lists for all PC mode observations, excluding those taken during orbit 2 which are affected by pile-up. The chosen 15 pixel source and 55 pixel background extraction regions are indicated by the small and large circles respectively. The total exposure time for the centre of this field is $430 \mathrm{ks} .99$ sources were detected at greater than $5 \sigma$.

region and at a radial distance that places it well outside of the location of the nearby bright star. Finally, increased X-ray flaring activity observed in the X-ray light-curve of the nearby star $1.8 \times 10^{6} \mathrm{~s}$ after the burst, forced us to use an even smaller 10 pixel wide extraction region, in order to minimise contamination of the XRT light-curve at the lowest count rates. Our chosen source and background regions (small and large circles respectively, together with a number of other sources detected in this field are shown in Fig. 1.

We note that the WT mode data taken during the first and second orbits falls upon the bad columns, which appeared following a micro-meteorite impact on 27th May 2005 (Abbey et al. 2005). Comparison of the XRT PSF with and without the bad columns shows that the WT data is affected at the $1.4 \%$ level. The WT mode data has been scaled accordingly. Finally, as mentioned earlier, the PC mode data taken during orbit 2 are affected by pile-up. To account for this we use an annular source extraction region of inner radius 4 pixels, and outer radius 15 pixels, for the 2nd orbit of data only.

\subsection{Combined BAT and XRT light-curve}

The combined BAT/XRT light-curve of GRB 051117A is shown in Fig. 2. The BAT light-curve has been constructed by extrapolating the BAT data into the $0.6-10 \mathrm{keV}$ XRT band, using the mean photon index of the combined fit to the BAT and WT mode XRT data in the $190 \mathrm{~s}$ overlap region. The XRT data were converted into flux units using the mean spectral fits to the $0.6-10 \mathrm{keV}$ band during the first orbit (WT mode data), 2nd orbit (PC mode data), and late-time PC mode data (orbit 3 onwards). For clarity the 1st orbit WT mode data have been binned to $>1000 \mathrm{ct} / \mathrm{bin}$. Orbit 2 (WT and PC mode data) data have been binned to $>100 \mathrm{ct} / \mathrm{bin}$. For orbit 3 and later, data have been binned to a minimum $20 \mathrm{ct} /$ bin at early times, and for $>3 \sigma$ detections at late times. The estimated mean background count rate over $\approx 400 \mathrm{ks}$ of observations within a 15 pixel radius circular aperture is $3.76 \pm 0.08 \times 10^{-4} \mathrm{ct} \mathrm{s}^{-1}$. An extrapolation of the late-time BAT light-curve appears to join smoothly with the XRT data taken at the beginning of orbit 3, suggesting that the 


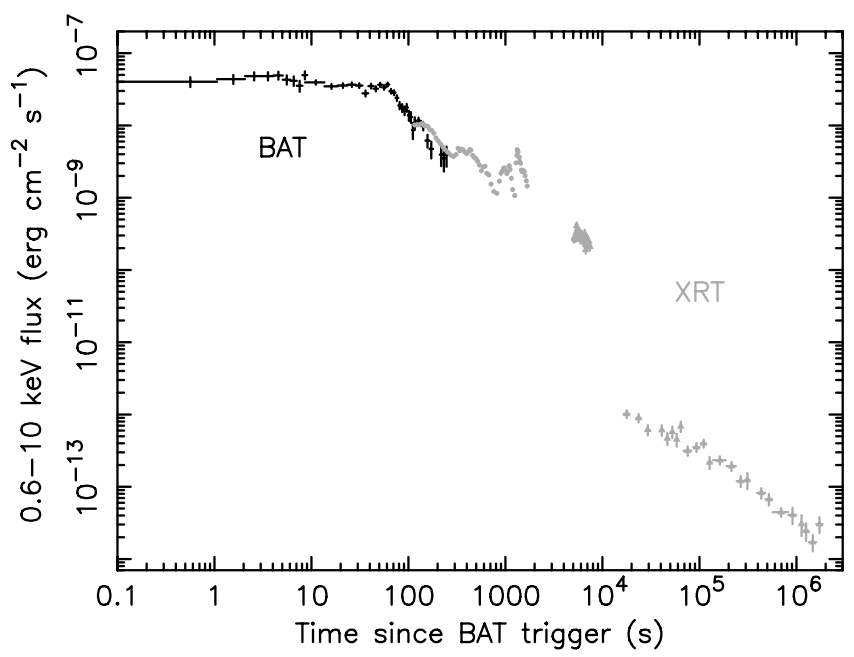

Fig. 2. The combined BAT 15-350 keV mask-weighted light-curve and XRT WT mode (orbits 1 and 2) and PC mode (orbits 2 and later) light-curve. The BAT count-rate light-curve has been converted into the 0.6-10.0 keV bandpass using the combined spectral fit to the BAT-XRT data taken in the overlap region. Similarly XRT count rate data from orbits 2 and later have been converted into flux units using the mean spectral fits to the XRT data taken during these 2 epochs.

flaring activity may have lasted at least until the end of the orbit 2 observations ( $7.5 \mathrm{ks}$ after the BAT trigger).

The XRT count rate light-curve can be approximated by a series of broken powerlaws with superposed flares at early times. The initial decline appears shallow, with an underlying slope of $\alpha=+0.77_{-0.06}^{+0.08}$, and displays numerous, relatively low amplitude short-lived flares. This relatively shallow early decay, suggests that the early X-ray light-curve behaviour is dominated by flaring activity. Indeed a simple extrapolation of the late time BAT light-curve to the late time XRT light-curve requires an underlying decay slope of $t^{-3}$, more typical of the fast X-ray decay slopes observed in many GRBs at early times (e.g. Tagliaferri et al. 2005).

The light-curve breaks sharply for $T_{0}+7450<t<T_{0}+$ $16500 \mathrm{~s}$ with $\alpha>5$, though the exact time of the break is undefined, before flattening again, with a slope of $\alpha=0.66 \pm 0.09$. At late times, the light-curve breaks once more to a steeper slope with $\alpha=1.1_{-0.14}^{+0.16}$, at $t=T_{0}+168440_{-80350}^{+97360} \mathrm{~s}$. The break in the light-curve at late times is highly significant. A fit to the late time data using a broken powerlaw as opposed to a single powerlaw yields $\Delta \chi^{2}=18$ for 2 fewer degrees of freedom.

\subsection{Timing characteristics of the prompt $X$-ray light-curve}

The early X-ray light-curve of GRB 051117A is characterised by a moderately slow decline with random, superposed flares of varying strength and duration. Visually the shape of the flares, and in particular the last flare in the first orbit of data, appear consistent with a fast-rise followed by an exponential decay (FRED-like), a form commonly observed in the prompt gammaray emission of many GRBs including GRB 051117A. In Table 2 we give the results of fitting the $\mathrm{X}$-ray light-curve with a single powerlaw decay slope, with slope 0.77 with a series of superposed FRED-like components, indicating the rise times and e-folding times for each of the individual flares. Note that the powerlaw is required to match the WT and PC mode data taken during orbit 2 (see Fig. 2). A power spectral analysis of the first orbit XRT data confirms the shot-noise like nature of the

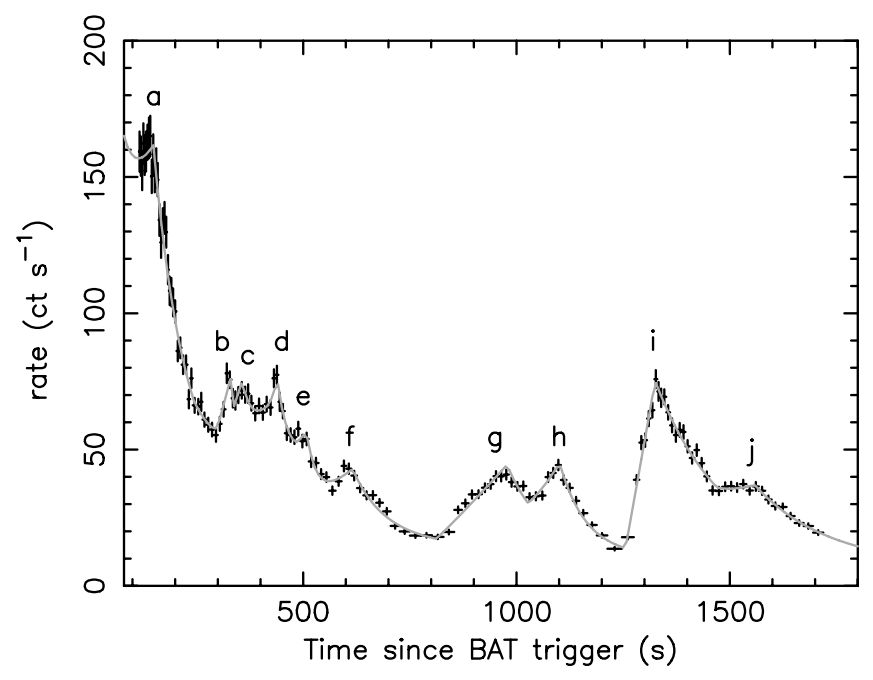

Fig. 3. Model fit to early X-ray light-curve. The light-curve is well-fit by a single powerlaw with slope -0.77 , with superposed FREDs (labelled $\mathrm{a}-\mathrm{j}$, see also Table 2 ) spanning a range in strength and duration, $\chi^{2}=$ 390 for 376.

Table 2. Parameterisation of the early X-ray light-curve. The underlying powerlaw has a slope of $\alpha=0.77_{-0.06}^{+0.08}$.

\begin{tabular}{rrrr}
\hline \hline flare no. & \multicolumn{1}{c}{$T_{\text {start }}$} & \multicolumn{1}{c}{$T_{\text {peak }}$} & e-folding timescale \\
$(\mathrm{s})$ & \multicolumn{1}{c}{$(\mathrm{s})$} & \multicolumn{1}{c}{$(\mathrm{s})$} \\
\hline $\mathrm{a}$ & 0 & $144_{-5}^{+6}$ & $80_{-2}^{+3}$ \\
$\mathrm{~b}$ & $298_{-7}^{+8}$ & $351_{-2}^{+10}$ & $47_{-29}^{+2}$ \\
$\mathrm{c}$ & $243_{-40}^{+32}$ & $331_{-6}^{+2}$ & $0.03_{-0.02}^{0.01}$ \\
$\mathrm{~d}$ & $417_{-18}^{+3}$ & $492_{-7}^{+14}$ & $101_{-7}^{+14}$ \\
$\mathrm{e}$ & $346_{-8}^{+10}$ & $441_{-6}^{+3}$ & $18_{-10}^{+10}$ \\
$\mathrm{f}$ & $572_{-8}^{+8}$ & $606_{-9}^{+5}$ & $68_{-20}^{+22}$ \\
$\mathrm{~g}$ & $810_{-8}^{+7}$ & $969_{-9}^{+9}$ & $53_{-7}^{+7}$ \\
$\mathrm{~h}$ & $978_{-27}^{+33}$ & $1112_{-4}^{+6}$ & $58_{-8}^{+6}$ \\
$\mathrm{i}$ & $1469_{-16}^{+14}$ & $1559_{-14}^{+20}$ & $171_{-56}^{+71}$ \\
$\mathrm{j}$ & $1255_{-2}^{+3}$ & $1325_{-3}^{+3}$ & $200_{-14}^{+15}$ \\
\hline
\end{tabular}

emission process (see e.g. Lehto 1989), with most of the power emitted on the longest timescales, and declining smoothly with a powerlaw slope of $\approx 2.6$, before flattening to the noise level on timescales shorter than $20 \mathrm{~s}$.

\section{The X-ray spectral properties of GRB 051117A}

Before Swift, prior evidence for excess absorption from GRB X-ray spectra is sketchy at best (see Stratta et al. 2004; De Luca et al. 2005; Gendre et al. 2005). More recently, in an analysis of 17 Swift detected GRBs (Campana et al. 2006a) found evidence for an observed column density above the Galactic value in $\sim 60 \%$ of cases. Campana et al. attribute the excess columns to an origin for long GRBs within dense molecular clouds (see also Galama \& Wijers 2001). We note that if the absorbing material is close to the GRB, the high radiation field can photo-ionise the surrounding material out to several parsecs, reducing the amount of low-energy aborption with time (Lazzati \& Perna 2002). The detection of excess Nh, variable or otherwise, is important as it provides strong supporting evidence for massive star progenitors and the association of long GRBs with starforming regions (Fruchter et al. 1999; Prochaska et al. 2004). Below we present a detailed spectral X-ray analysis of the early and late time X-ray specta for GRB 051117A. 


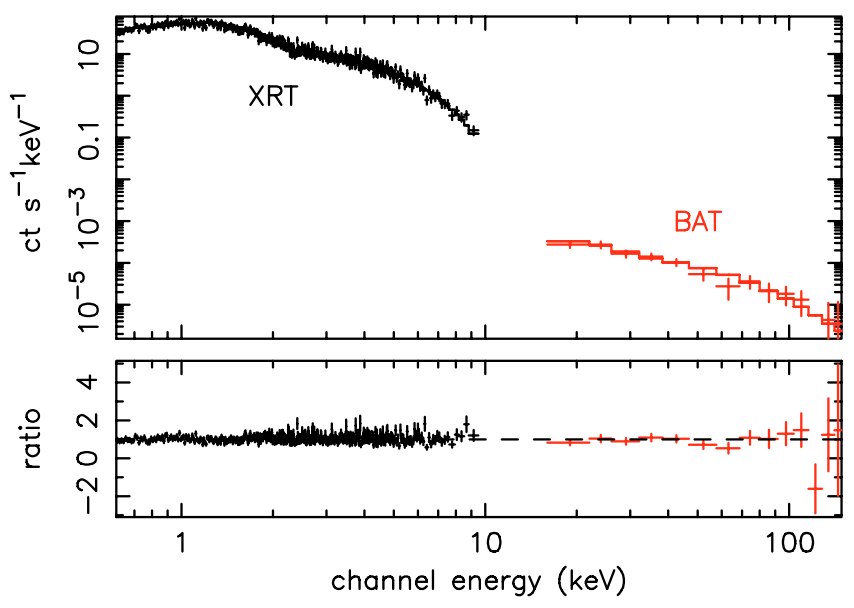

Fig. 4. A fit to the combined BAT-XRT spectrum covering the time interval $T_{0}+113 \mathrm{~s}$ to $T_{0}+303 \mathrm{~s}$. The data are well fit by a Band function, with $\mathrm{Nh}_{\text {excess }}=1.6 \pm 0.6 \times 10^{21} \mathrm{~cm}^{-2}, \alpha=-0.43_{-0.84}^{+0.3}, \beta=-1.95 \pm 0.04$, and $E_{\text {break }}=1.0_{-\infty}^{+0.7} \mathrm{keV}, \chi^{2}=414$ for 404 d.o.f.

\subsection{Joint BAT/XRT spectrum}

The long duration of this GRB provides a $190 \mathrm{~s}$ observation window (covering the time interval $T_{0}+113 \mathrm{~s}$ to $T_{0}+303 \mathrm{~s}$ ) for which we have significant counts in both the BAT and XRT. In Fig. 4 we show the joint BAT/XRT spectrum extracted over this time interval. The joint BAT/XRT spectrum can be well fit with a single absorbed powerlaw with $\mathrm{Nh}_{\text {excess }}=2.3 \times 10^{21} \mathrm{~cm}^{-2}$, photon index $\Gamma=2.0, \chi^{2}=421$ for 409 d.o.f. A Band function (Band et al. 1993) with excess absorption, also provides an acceptable fit to the data, with $\mathrm{Nh}_{\text {excess }}=1.6 \pm 0.6 \times 10^{21} \mathrm{~cm}^{-2}$, $\alpha=-0.43_{-0.84}^{+0.3}, \beta=-1.95 \pm 0.04$, and $E_{\text {break }}=1.0_{-\infty}^{+0.7} \mathrm{keV}$, $\chi^{2}=414$ for 404 d.o.f. We note that while excess $\mathrm{Nh}$ is required by the Band function model fit $\left(\Delta \chi^{2}=52\right.$ for one less d.o.f.), we obtain only an upper limit to the break energy with this model.

\subsection{The prompt $X$-ray spectrum}

The high observed count rate and slow rate of decline during the first orbit of XRT WT mode data, allows us to construct amongst the highest quality $\mathrm{S} / \mathrm{N}$ spectrum of any burst yet observed by Swift comparable to the bright bursts GRB 061121 (Page et al. 2006) and GRB 060124 (Holland et al. 2006). In Fig. 5 we show a model fit to the mean WT mode spectrum accumulated during the first orbit (1605 s of data). Fitting a single absorbed powerlaw to the $0.6-10.0 \mathrm{keV}$ band, with $\mathrm{Nh}$ tied at the Galactic value of $1.82 \times 10^{20} \mathrm{~cm}^{-2}$ is a very poor description of the data, $\chi^{2}=2777$ for 534 d.o.f. Allowing for excess Nh provides a significant improvement, with a best-fit model of $\Gamma=2.23 \pm 0.02$, $\mathrm{Nh}_{\text {excess }}=2.11 \pm 0.01 \times 10^{21} \mathrm{~cm}^{-2}$, with $\chi^{2}=523$ for $533 \mathrm{de}-$ grees of freedom, all errors $90 \%$ confidence on 1 interesting parameter. An absorbed broken powerlaw model, with $\mathrm{Nh}$ above the Galactic value, provides an improved fit to the data, with a best-fit $\Gamma_{1}=1.77 \pm 0.23, \Gamma_{2}=2.19 \pm 0.03$, and break energy $E_{\text {break }}=1.04_{-0.13}^{+0.08}, \chi^{2}=511$ for 531 d.o.f.

We have searched for the possible existence of emissionlines in the WT mode spectrum taken during the first orbit of observations. At near the peak of the effective area $(1 \mathrm{keV})$, we can rule out the presence of narrow spectral features $(\sigma=100 \mathrm{eV})$ with $E W$ less than $15 \mathrm{eV}$ at greater than $3 \sigma$ confidence. Broader spectral features cannot be excluded by the data, due to uncertainties in the spectral calibration.

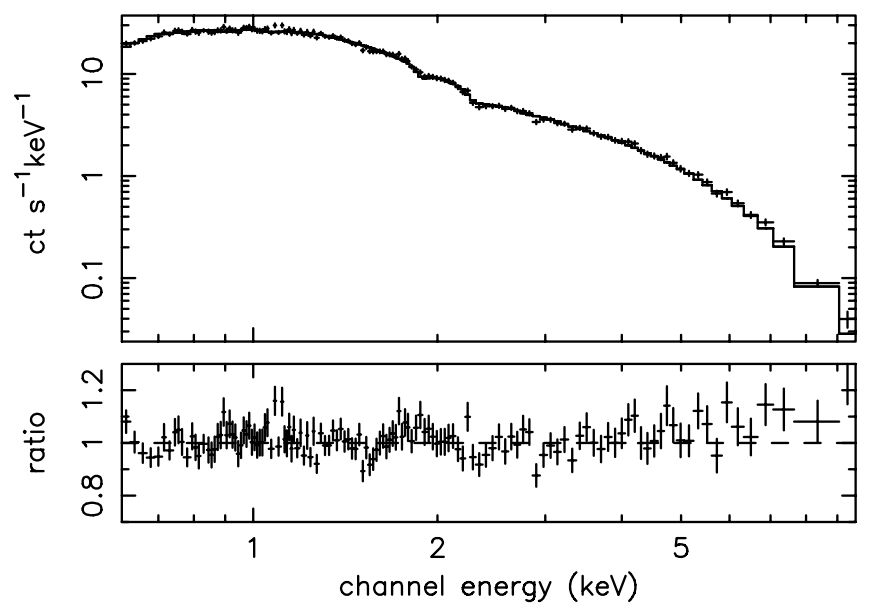

Fig. 5. WT mode spectrum formed from the first orbit of WT mode data (1605 s). The spectrum is best fit by an absorbed broken powerlaw model, with $\mathrm{Nh}$ above the Galactic value, with $\Gamma_{1}=1.77 \pm 0.23$, $\Gamma_{2}=2.19 \pm 0.03$, and break energy $E_{\text {break }}=1.04_{-0.13}^{+0.08}$ (all errors $90 \%$ confidence), $\chi^{2}=511$ for 531 d.o.f. (see text for details).

\subsection{Global spectral properties}

To investigate the long term spectral evolution of the prompt and early afterglow emission of GRB 051117A, we have formed average spectra (see Fig. 6) covering the 1st orbit (WT data only, $1605 \mathrm{~s}), 2$ nd orbit [(both WT (163 s) and PC mode data (2313 s)] and the late time PC mode data (orbit 3 onwards, $411 \mathrm{ks}$ ). A single absorbed power-law fit to each of the four spectra with excess $\mathrm{Nh}$ above the Galactic value of $1.82 \times 10^{20} \mathrm{~cm}^{-2}$ is a good description of the data, with $\Gamma=2.24 \pm 0.02, \mathrm{Nh}_{\text {excess }}=$ $2.1 \times 10^{21} \mathrm{~cm}^{-2}, \chi^{2}=708$ for 679 d.o.f. Untying $\Gamma$ between each of the 4 segments, provides a significant improvement, $\Delta \chi^{2}=42$, F-statistic 14.3 , null hypothesis $4.7 \times 10^{-9}$. We note that keeping $\Gamma$ tied and untying Nh provides an equally good description of the data, though excess $\mathrm{Nh}$ is consistent with being constant within the errors.

Finally, we tried to fit an absorbed broken powerlaw to each of the segments, with $\Gamma$ tied pre- and post-break and excess $\mathrm{Nh}$ of $1.3 \times 10^{21} \mathrm{~cm}^{-2}$ tied between the segments. This model is only marginally worse, with $\chi^{2}=669$ for 674 d.o.f. Untying $\Gamma$ between the spectra, significantly improves the fit $\chi^{2}=645$ for 668 d.o.f. (see Table 3 for details). With such good quality data, the lack of evidence for a variable Nh suggests that the absorbing column may not be associated with the immediate GRB environment.

\subsection{Spectral characteristics of the early $X$-ray light-curve}

The superb quality, i.e. high observed count rate and relatively slow temporal decline, exhibited by the early X-ray light-curve of GRB 051117A during the first orbit affords a detailed temporal analysis of the X-ray spectral evolution for this source. For this analysis, the early X-ray light-curve was divided into segments containing approximately $2000 \mathrm{ct} \mathrm{bin}{ }^{-1}$, and spectra extracted for each segment. This binning scheme represents the best compromise between spectra with approximately equivalent signal-to-noise, and high temporal resolution, and allows us to investigate the X-ray spectral evolution of this source in a uniform manner. The binned light-curve composed of 37 segments of data is shown in Fig. 7 (top panel). The early XRT light curve is characterised by a relatively slow temporal decline $(\alpha=0.77)$ 
Table 3. Swift XRT spectral fits. Quoted errors are $90 \%$ confidence on 1 interesting parameter.

\begin{tabular}{|c|c|c|c|c|}
\hline \multirow[b]{2}{*}{$\begin{array}{l}\text { Mode } \\
\Gamma \\
\chi_{v}^{2} / \text { d.o.f. }\end{array}$} & \multicolumn{4}{|c|}{$\begin{array}{c}\text { GRB 0501117A } \\
\text { Model 1: } w a *\left(w a * p o^{\dagger}\right)-\mathrm{Nh}_{\mathrm{Gal}}=1.82 \times 10^{20} \mathrm{~cm}^{-2}, \mathrm{Nh}_{\text {excess }}=2.1 \times 10^{21} \mathrm{~cm}^{-2}, \Gamma \text { untied }\end{array}$} \\
\hline & $\begin{array}{c}\text { WT orb } 1 \\
2.23 \pm 0.02 \\
666 / 676\end{array}$ & $\begin{array}{l}\text { WT orb } 2 \\
2.39_{-0.15}^{+0.16}\end{array}$ & $\begin{array}{l}\text { PC orb } 2 \\
2.45_{-0.07}^{+0.08}\end{array}$ & $\begin{array}{l}\text { PC orb } 3 \text { onward } \\
2.52 \pm_{-0.08}^{+0.15}\end{array}$ \\
\hline & \multicolumn{4}{|c|}{ Model 2: $w a *(w a * p o)-\Gamma=2.24, \mathrm{Nh}_{\mathrm{Gal}}=1.82 \times 10^{20} \mathrm{~cm}^{-2}, \mathrm{Nh}_{\text {excess }}$ untied } \\
\hline $\begin{array}{l}\text { Mode } \\
N_{\text {excess }} \\
\chi_{v}^{2} / \text { d.o.f. }\end{array}$ & $\begin{array}{l}\text { WT orb } 1 \\
0.21 \\
664 / 676\end{array}$ & $\begin{array}{l}\text { WT orb } 2 \\
0.17\end{array}$ & $\begin{array}{l}\mathrm{PC} \text { orb } 2 \\
0.12\end{array}$ & $\begin{array}{l}\text { PC orb } 3 \text { onward } \\
\quad 0.11\end{array}$ \\
\hline & \multicolumn{4}{|c|}{$\begin{array}{l}\text { Model 3: } w a *(\text { wa } * \text { bknpow })-\Gamma-\text { pre }=1.53 \pm 0.2, \Gamma-\text { post }=2.17 \pm 0.03 \\
\quad \text { and tied, } \mathrm{Nh}_{\mathrm{Gal}}=1.82 \times 10^{20} \mathrm{~cm}^{-2}, \mathrm{Nh}_{\text {excess }}=1.32 \pm 0.02 \times 10^{21} \mathrm{~cm}^{-2}\end{array}$} \\
\hline $\begin{array}{c}\text { Mode } \\
E_{\text {break }} \\
\chi_{v}^{2}\end{array}$ & $\begin{array}{c}\text { WT orb } 1 \\
1.10 \pm 0.06 \\
669 / 674\end{array}$ & $\begin{array}{l}\text { WT orb } 2 \\
0.95_{-\infty}^{+0.30}\end{array}$ & $\begin{array}{l}\text { PC orb } 2 \\
0.56_{-\infty}^{+0.26}\end{array}$ & $\begin{array}{l}\text { PC orb } 3 \text { onward } \\
0.60_{-\infty}^{+0.08}\end{array}$ \\
\hline & \multicolumn{4}{|c|}{$\begin{array}{c}\text { Model 4: wa } *(\text { wa } * \text { bknpow }) \\
\Gamma \text { untied, } \mathrm{Nh}_{\mathrm{Gal}}=1.82 \times 10^{20} \mathrm{~cm}^{-2}, \mathrm{Nh}_{\text {excess }}=1.32 \pm 0.02 \times 10^{21} \mathrm{~cm}^{-2}\end{array}$} \\
\hline $\begin{array}{c}\text { Mode } \\
E_{\text {break }} \\
\chi_{v}^{2}\end{array}$ & $\begin{array}{l}\text { WT orb } 1 \\
1.10 \pm 0.06 \\
669 / 674\end{array}$ & $\begin{array}{l}\text { WT orb } 2 \\
0.95_{-\infty}^{+0.30}\end{array}$ & $\begin{array}{l}\text { PC orb } 2 \\
0.56_{-\infty}^{+0.26}\end{array}$ & $\begin{array}{l}\text { PC orb } 3 \text { onward } \\
0.60_{-\infty}^{+0.08}\end{array}$ \\
\hline
\end{tabular}

$\dagger$ Here we use the standard XSPEC notation where $w a *$ po means absorbed powerlaw.

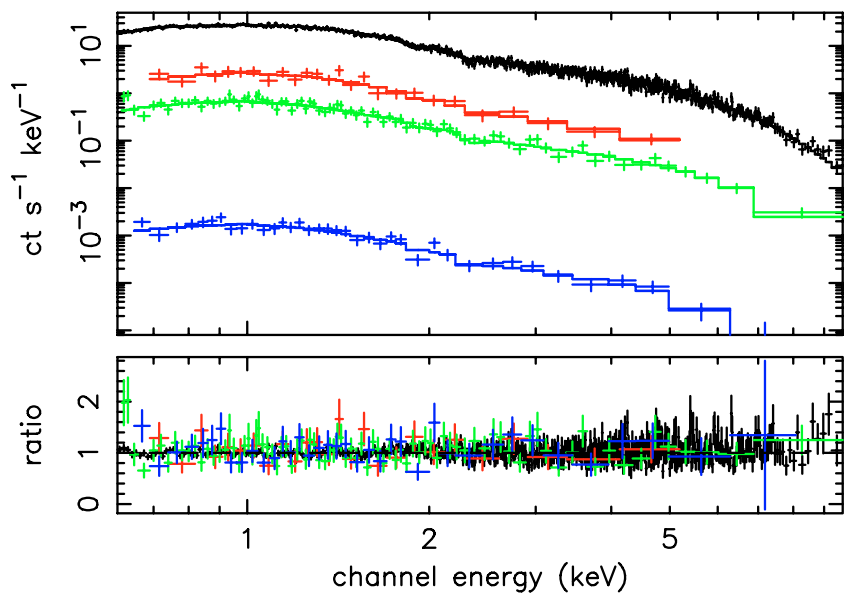

Fig. 6. WT mode (black and red, 1st and 2nd orbit respectively), PC mode (green 2nd orbit only, blue orbit 3 and later) spectra $(0.6-10 \mathrm{keV})$ for GRB 051117A. The data are well fit by an absorbed powerlaw, with an excess $\mathrm{Nh}$ above the Galactic value of $1.82 \times 10^{20} \mathrm{~cm}^{-2}$ of $2.1 \times$ $10^{21} \mathrm{~cm}^{-2}$, and $\Gamma$ increasing through each of the 4 segments, $\chi^{2}=666$ for 676 d.o.f.

with superposed flares of varying strength and duration. For each of the 37 segments of WT mode data we extract spectra from the cleaned event list, using the standard grade selection (grade 0-2 for WT mode), and using a 30 pixel wide window centred on the source position.

A simultaneous fit to the 37 spectra over the $0.6-10.0 \mathrm{keV}$ band, using a simple absorbed powerlaw, with $\mathrm{Nh}$ fixed at the Galactic value of $1.82 \times 10^{20} \mathrm{~cm}^{-2}$, excess absorption of $2.1 \times 10^{21} \mathrm{~cm}^{-2}$ with $\Gamma$ and the normalisation untied between segments, provides an acceptable fit $\chi^{2}=2470$ for 2648 d.o.f. Figure 7 (middle panel) shows the derived photon indices for each time segment (all errors 90\% confidence). Similarly, an absorbed broken powerlaw fit to the spectra, with Nh fixed at the Galactic value, the photon index pre-break tied together, the photon index post-break tied together, and the break energy $E_{\text {break }}$ and normalisation untied, also provides an acceptable fit to the data $\chi^{2}=2593$ for 2647 d.o.f., without requiring excess $\mathrm{Nh}$. Including excess Nh only moderately improves the fit $\Delta \chi^{2}=22$. The variation in break energy for each of the 37 segments for the broken powerlaw model without excess $\mathrm{Nh}$ as a function of time is shown in Fig. 7 (lower panel). We checked for the presence of variable excess NH in our data, by allowing Nh to freely vary between segments. However, although there is clear evidence for variable $\Gamma$, we find no evidence for variable Nh. Figure 8 shows the $68 \%, 95 \%$ and $99 \%$ confidence contours for $\Gamma$ versus $\mathrm{Nh}$ taken from 3 data segments spanning the full range in source count rate ${ }^{2}$.

Visual inspection of Fig. 7, panels 1 and 2, shows that there exists a strong anti-correlation between the source intensity and photon index, such that the spectrum is much harder when the source is brighter. This can be seen more clearly in Fig. 9 where we plot the hardness, HR, here defined as the ratio of the counts in the $2.0-10.0 \mathrm{keV}$ band relative to the $0.2-2.0 \mathrm{keV}$ band, as a function of time. Source intensity and hardness ratio are found to be highly correlated with a Pearson's linear correlation coefficient $r=0.85$ with a probability $P$ of no correlation which is vanishingly small. In Fig. 10 we show the source intensity $(0.6-10 \mathrm{keV})$ as a function of photon index $\Gamma$. This again shows the source is spectrally harder when brighter, though we note that the exact correspondence between the two parameters is not simply one to one. Moreover, the variation in $\Gamma$ with source intensity appears to shows signs of hysteresis. That is, the variation in $\Gamma$ with source intensity for each flare, appears to follow similar tracks. The observed spectral behaviour is entirely consistent with the gamma-ray spectral evolution seen in BATSE GRBs (e.g. Ford et al. 1995; Bhat et al. 1994).

Both Fig. 7 (middle panel) and Fig. 9, indicate that the hardening of the spectrum coincides with each new flaring episode.

\footnotetext{
2 We have checked that temperature dependent variable gain and bias offsets, which can produce $\sim 20 \mathrm{eV}$ shifts in the energy scale do not effect our conclusions.
} 


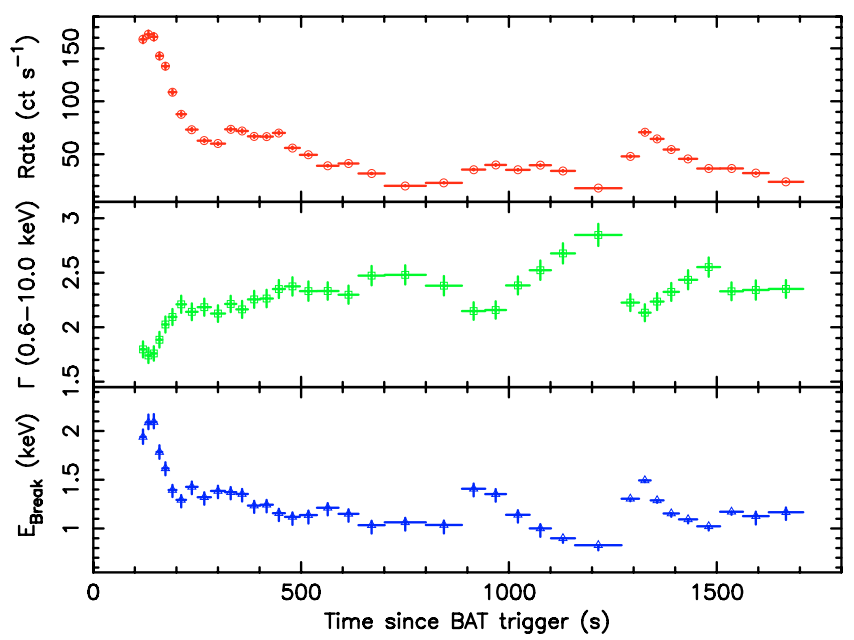

Fig. 7. Upper panel - The $0.6-10.0 \mathrm{keV}$ WT mode light-curve of the 1 st orbit data, binned to $2000 \mathrm{ct} \mathrm{bin}^{-1}$. Middle panel - The temporal variation in photon index $\Gamma$, derived from a simultaneous fit to $37 \mathrm{spec}-$ tra, assuming a simple absorbed powerlaw, with excess $\mathrm{Nh}$ tied between each of the segments and $\Gamma$ allowed to freely vary. Lower panel - The variation in break energy, $E_{\text {break }}$, for the same spectra, adopting a broken powerlaw spectral model with $\Gamma$ pre- and post-break tied between the spectra. In this model excess $\mathrm{Nh}$ is not included (see text for details).

The spectrum is then observed to soften gradually (this can be seen either in terms of an increase in the Photon index or a decrease in HR), as the X-ray intensity fades (see also Fig. 10). The observed strong correlation between source intensity and spectral shape suggests a common origin for each of the flares. The simplest mechanism which can account for the observed temporal behaviour, is one in which the overall spectral shape remains constant, but the characteristic break energy $E_{\text {break }}$ varies with source intensity (see also Crider et al. 1997). Over the limited band-pass of the XRT, a small increase in the break-energy will harden the spectrum, while a small decrease in break-energy will soften the spectrum. In Fig. 11 we show a cartoon illustrating this behaviour, and (inset) the resultant variation in photon index $\Gamma$. In Fig. 12 we show the model fits to the X-ray spectra for each of the segments covering the last flare (segments 28-37). We note that the peak of the Band function fit $(1 \mathrm{keV})$ to the joint BAT-XRT data taken during the time interval $T_{0}+113 \mathrm{~s}<t<T_{0}+303 \mathrm{~s}$ does indeed lie within the XRT bandpass, adding strong support to this simple picture.

\section{Observations of GRB 051117A in other bands}

Swift UVOT began observing GRB 051117A at 10:53:10 UT, $111 \mathrm{~s}$ after the BAT trigger. A faint uncatalogued source was detected in a $200 \mathrm{~s} V$-band image, with a magnitude of $V=$ $20.01 \pm 0.46(1-\sigma$, statistical, uncorrected $)$. The extinction in this direction is $A_{V}=0.08$ (Band et al. 2005; Holland et al. 2005). In a $50 \mathrm{~s}$ UVOT white-light observation starting $531 \mathrm{~s}$ after the BAT trigger the source had a magnitude of $19.19 \pm 0.16$ (1- $\sigma$, statistical). 3.5 days after the burst the source had faded to a white-light magnitude of approximately 21.5 (see Fig. 14 and Table 4 for a detailed description of the UVOT observations). Interestingly, there is weak evidence that the source is extended, and which may be associated with the host galaxy of GRB 051117A. During the fast decay observed in XRT $\approx 10^{4} \mathrm{~s}$ after the burst, the $V$-band magnitude remained remarkably constant. The optical flux declines rapidly (as seen in the white light filter) $3 \times 10^{4} \mathrm{~s}$ after the BAT trigger.

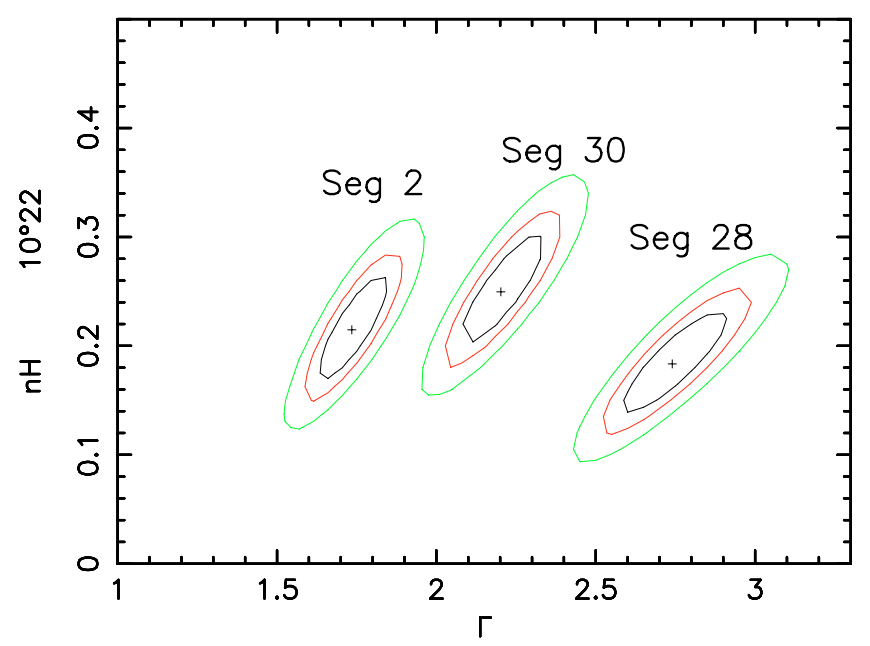

Fig. 8. Variation of excess $\mathrm{Nh}$ and photon index $\Gamma$ for segments 2, 28 and 30 .

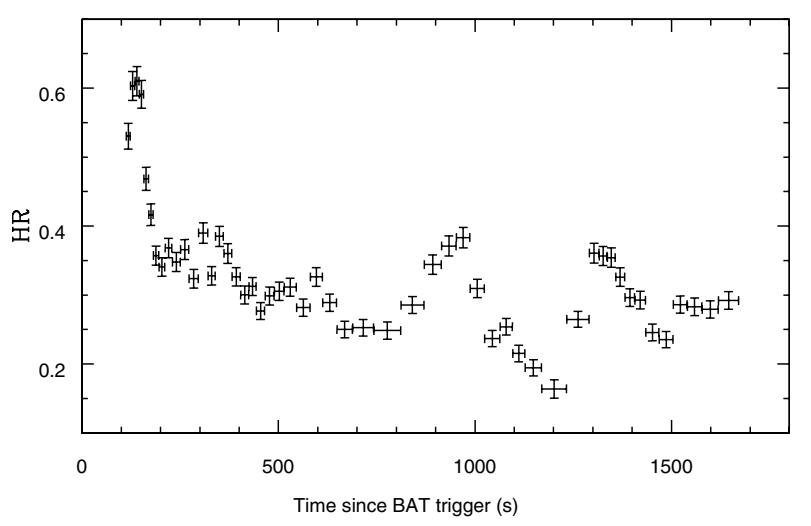

Fig. 9. Hardness ratio $H R=H / S$, where $H R$ is the ratio of counts in the $2.0-10.0 / 0.2-2.0 \mathrm{keV}$ band.

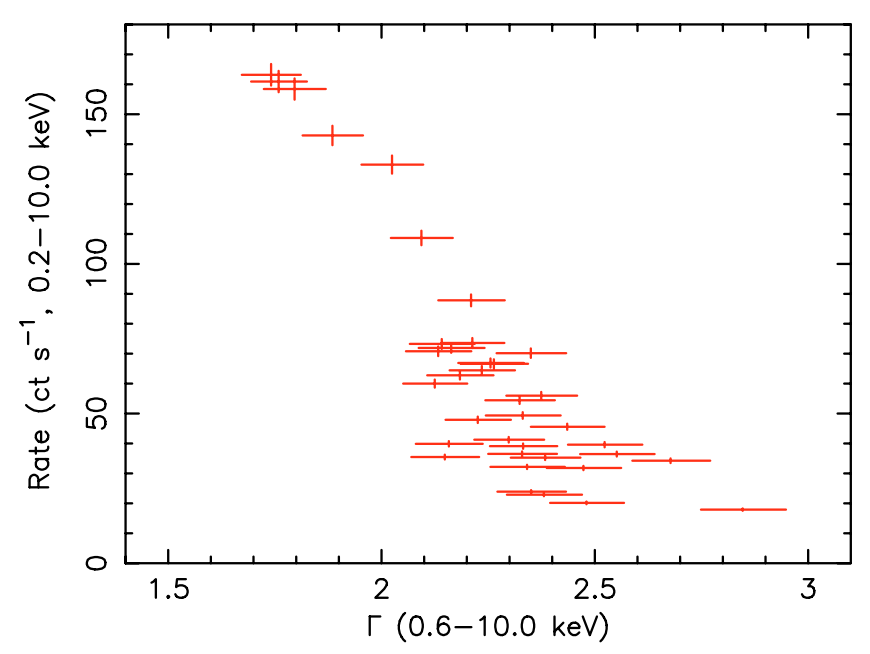

Fig. 10. Photon index as a function of count-rate. This confirms that the spectra are harder when brighter.

In the interval $1000 \mathrm{~s}$ to $300 \mathrm{ks}$ after the BAT trigger, the source intensity (in both $V$ and White light) decreased by 3 magnitudes, equivalent to a powerlaw decay rate of $\alpha=0.5$. Since this interval covers the sharp break in the XRT light-curve, the optical light-curve has a much shallower decay than the $\mathrm{X}$-ray light-curve. We compiled a spectral energy distribution for the UVOT and XRT data at $750 \mathrm{~s}$ after the BAT trigger to 


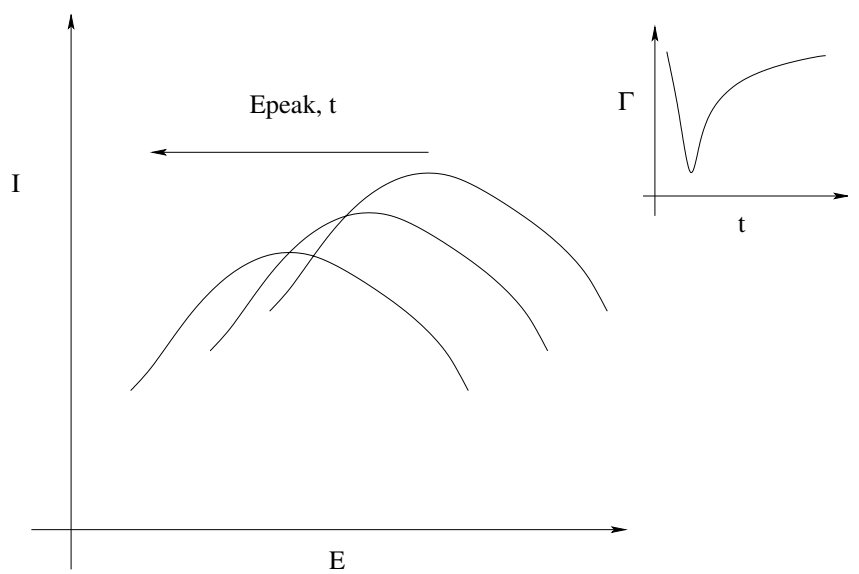

Fig. 11. Cartoon model indicating the variation in photon index $\Gamma$ (inset), for a spectrum consisting of a broken powerlaw, as the breakenergy moves to lower energies.

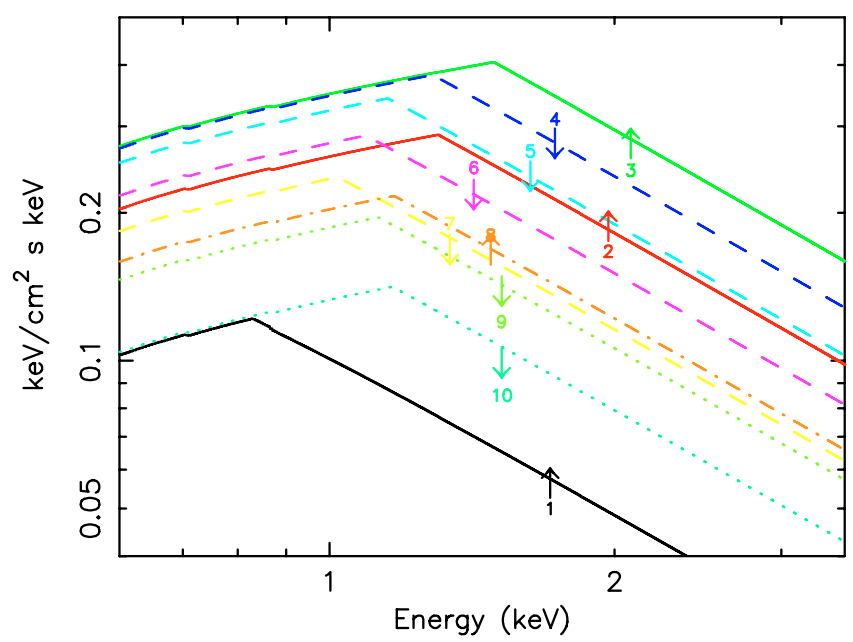

Fig. 12. Model fits to the X-ray spectra extracted from the last observed flaring episode of first orbit of data (10 spectra of approx $2000 \mathrm{ct}$, numbered sequentially from 1-10). Here we model the data as an absorbed broken powerlaw, with $\Gamma$ pre-break tied together, $\Gamma$ post-break tied together, and excess Nh tied together. The general trend is for an increase in break energy as the source intensity increases (fits 1-3), and a decrease in break energy as the source intensity decreases. We note however, that while clearly correlated, there is no one-one correspondence between break-energy and source intensity.

investigate absorption and spectral shape in the UV and optical data (Fig. 13). Inclusion of the XRT data ensures a more accurate fit to the underlying power law, but we note that near infrared data, not available for this source, are also required for a precise determination of the underlying continuum. We find that a single power law cannot adequately fit the SED, and a broken power law is required. We obtain a good fit with a pre-break slope of $\Gamma \sim 1$, breaking in between the optical and X-ray data to $\Gamma=2.20 \pm 0.16$, consistent with the slope measured from the $\mathrm{X}$-ray data alone. This spectral break cannot be identified as a cooling break due to the large errors on the optical/UV spectral slope, but we note that in the case of a cooling break $\beta_{2}=\beta_{1}+\frac{1}{2}$ (where $\beta=\Gamma-1$ ) which is not found in the best-fitting model to this SED.

Assuming a redshift of $z=0.73$, as estimated in Sect. 5.1, we clearly detect excess absorption in both the optical and the $\mathrm{X}$-ray regimes. We find $E(B-V) \sim 0.12$ for either a Large or

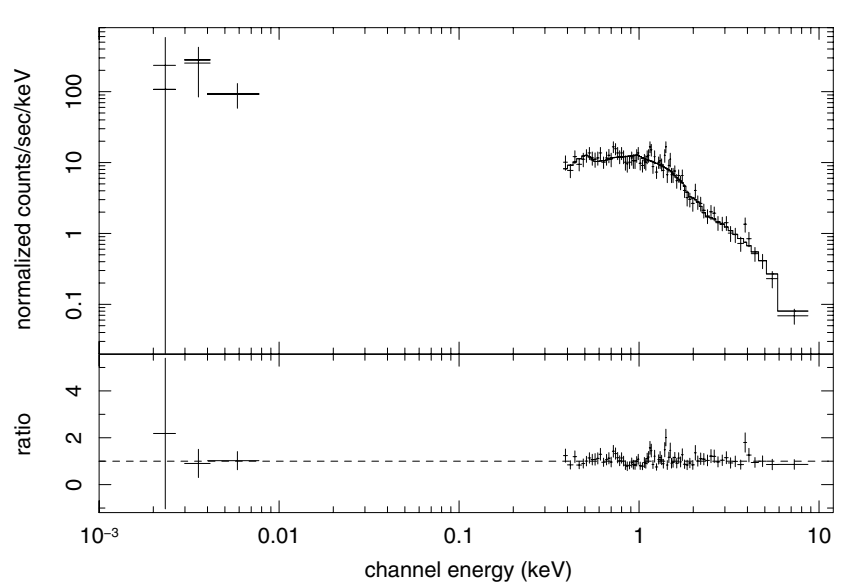

Fig. 13. Broad-band spectral energy distribution (SED) of GRB 051117A. This SED was compiled at a logarithmic midpoint time of $750 \mathrm{~s}$ after the BAT trigger (see text for details).

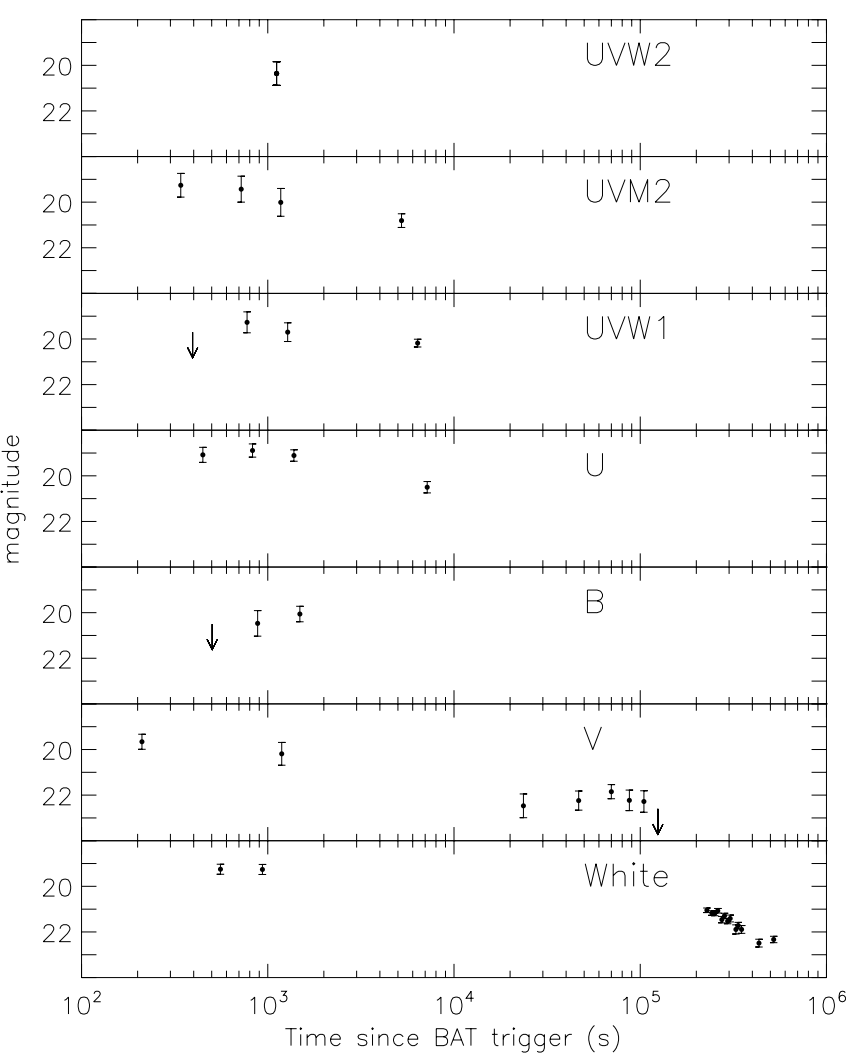

Fig. 14. UVOT ultraviolet ( $U W 2, U V M 2, U W 1)$, and optical $(U, B, V$, and white) light-curves of GRB 051117A.

Small Magellanic Cloud extinction law (Pei 1992) and $N_{\mathrm{H}} \sim$ $1.5 \times 10^{21} \mathrm{~cm}^{-2}$, again consistent with fits to the X-ray data alone.

Radio observations starting approximately 8 hours after the BAT trigger, with the Very Large Array operating at $8.5 \mathrm{GHz}$ did not detect an afterglow at greater than 3- $\sigma$, for an rms noise level of $56 \mu \mathrm{Jy}$ (Frail, D. 2005, GCN 4292).

\section{Discussion}

The standard afterglow model of a spherical blast wave expanding uniformly into a spherically symmetric ambient medium with a smooth density profile, predicts smooth afterglow 
Table 4. UVOT multicolour photometry.

\begin{tabular}{rrcccr}
\hline \hline$T_{\text {mid }}$ & $\begin{array}{r}\text { Exposure } \\
(\mathrm{s})\end{array}$ & Filter & Mag & Err & Significance \\
\hline 503 & 50 & $B$ & $>20.5$ & - & 2.0 \\
882 & 50 & $B$ & 20.47 & 0.56 & 2.0 \\
1487 & 100 & $B$ & 20.06 & 0.34 & 3.4 \\
341 & 50 & $U V M 2$ & 19.26 & 0.52 & 2.2 \\
720 & 50 & $U V M 2$ & 19.43 & 0.57 & 2.1 \\
1174 & 100 & $U V M 2$ & 20.01 & 0.61 & 2.3 \\
5227 & 841 & $U V M 2$ & 20.81 & 0.30 & 3.7 \\
448 & 50 & $U$ & 19.08 & 0.33 & 3.6 \\
828 & 50 & $U$ & 18.89 & 0.29 & 4.0 \\
1382 & 100 & $U$ & 19.11 & 0.25 & 4.6 \\
7178 & 686 & $U$ & 20.50 & 0.25 & 4.4 \\
211 & 200 & $V$ & 19.66 & 0.33 & 3.4 \\
1188 & 239 & $V$ & 20.19 & 0.50 & 2.2 \\
395 & 50 & $U V W 1$ & $>19.7$ & - & 2.0 \\
774 & 50 & $U V W 1$ & 19.27 & 0.46 & 2.5 \\
1279 & 100 & $U V W 1$ & 19.70 & 0.41 & 2.7 \\
6378 & 900 & $U V W 1$ & 20.18 & 0.17 & 6.4 \\
1115 & 199 & $U V W 2$ & 20.36 & 0.52 & 2.3 \\
557 & 50 & White & 19.25 & 0.22 & 5.5 \\
936 & 50 & White & 19.26 & 0.22 & 5.6 \\
23591 & 7673 & $V$ & 22.47 & 0.52 & 2.2 \\
46705 & 7694 & $V$ & 22.24 & 0.42 & 2.6 \\
69961 & 6523 & $V$ & 21.85 & 0.31 & 3.6 \\
87410 & 6307 & $V$ & 22.23 & 0.45 & 2.5 \\
104641 & 6872 & $V$ & 22.28 & 0.47 & 2.4 \\
124381 & 8197 & $V$ & $>22.6$ & - & 2.0 \\
228052 & 4957 & White & 21.05 & 0.10 & 12.0 \\
242588 & 4995 & White & 21.17 & 0.10 & 11.7 \\
251297 & 7225 & White & 21.19 & 0.10 & 13.6 \\
260713 & 5277 & White & 21.07 & 0.10 & 13.2 \\
274342 & 4901 & White & 21.46 & 0.14 & 8.7 \\
283480 & 5916 & White & 21.29 & 0.11 & 11.3 \\
294677 & 7159 & White & 21.53 & 0.11 & 10.4 \\
304093 & 3817 & White & 21.41 & 0.15 & 7.9 \\
326447 & 5026 & White & 21.89 & 0.20 & 5.8 \\
335930 & 7276 & White & 21.72 & 0.14 & 8.7 \\
350046 & 6887 & White & 21.89 & 0.17 & 7.1 \\
434098 & 20598 & White & 22.49 & 0.17 & 6.9 \\
520639 & 21926 & White & 22.33 & 0.14 & 8.4 \\
\hline & & & & &
\end{tabular}

light-curves. By contrast the early X-ray decay light-curve of GRB 051117A shows a highly complex structure which can be modelled as a superposition of FRED-like shots on a smooth underlying powerlaw decay. Complex flaring behaviour in the early X-ray light-curve appears to be a relatively common feature, being seen in approximately half of all GRBs observed by Swift (Burrows et al. 2005a; Falcone et al. 2006). Suggested origins for the appearance of flares include: (i) density fluctuations in the medium into which the blast-wave expands, (ii) patchy shells, (iii) refreshed shocks, and (iv) late time engine activity (Ioka et al. 2005). As noted by Ioka et al. (2005), the relative fluctuation amplitudes $\left(\Delta F_{v} / F_{v}\right)$ and timescales $(\Delta t / t)$ of individual flares can be used to rule out some of these models. For GRB 051117A, even the longest flares have timing properties which are inconsistent with refreshed shocks $(\Delta t / t<1 / 4)$. The patchy shell model also fails, as $\Delta t<t$ in all cases. While the low-amplitude flares have timescales $(\Delta t / t \ll 0.01)$ which are marginally consistent with an origin due to small-scale density fluctuations in the ambient medium (in the off-axis case), density fluctuations cannot explain either the observed shape of the flares (flares due to density enhancements are typically characterised by a slow rise to peak, e.g. Nakar \& Granot 2006) or the origin of the longer duration flares. Furthermore, invoking two distinct mechanisms for flaring behaviour for flares which, in all other respects, exhibit similar spectral and timing, characteristics, is somewhat unsatisfactory. Though it is possible that a variety of mechanisms may be operating at the same time, the simplest explanation is that the observed variations are in fact indicative of late time engine activity in this burst (see also Lazzati \& Perna (2007) for a full discussion on the origin of flares in GRBs, and Guetta et al. (2006) for an in depth analysis of X-ray flaring activity in Swift GRB observations.). The close agreement between the BAT and XRT light-curves in the overlap region, and the apparent connection of the late-time BAT light-curve and XRT light-curve from orbit 3, strongly supports this hypothesis. Moreover, the power spectrum of the early Xray light-curve, after detrending the data to remove the underlying steep decay, is consistent with a shot noise process, which implies by definition a range of amplitudes and timescales for individual flares (see Fig. 3, and Table 2).

If the flaring activity is indeed related to late-time energy injection (i.e. continued activity of the central engine), then for GRB 051117A the engine possibly remains active for far longer than previously supposed. The steep decline for times $T_{0}+7450<t<T_{0}+16500$ is most-likely due to the curvature effect. If so, then this represents amongst the latest detections of the curvature effect (even after allowing for correction to the rest-frame) of any GRB, comparable to that observed in GRB 050904 (Cummings et al. 2005), and GRB 070110 (Krimm et al. 2007).

\subsection{Redshift and luminosity}

For GRB 051117A there is currently no redshift estimate for the host galaxy. However, we can estimate the redshift using the empirical relationship $\left|\Gamma_{\gamma}\right|=(2.76 \pm 0.09)(1+z)^{-0.75 \pm 0.06}$ (Amati et al. 2002). For GRB 051117A, $\Gamma_{\gamma}=1.83 \pm 0.07$ $(15.0-150 \mathrm{keV})$, we estimate a redshift of $z=0.73_{-0.21}^{+0.17}$. This redshift is consistent with the upper limit placed on $\mathrm{z}$ from the strong detection of this GRB in all of the UVOT filters $(z<1.4$, adopting $\lambda 2200 \AA$ as the central wavelength for UVW2). For a BAT fluence of $4.6 \times 10^{-6} \mathrm{erg} \mathrm{cm}^{-2}$ we estimate an isotropic gamma-ray energy of $E_{\text {iso }}=7.8 \times 10^{51} \mathrm{erg}$ (assuming a WMAP Cosmology of $H_{0}=70 \mathrm{~km} \mathrm{~s}^{-1} \mathrm{Mpc}^{-1}, \Omega_{\lambda}=0.73, \Omega_{m}=1-\Omega_{\lambda}$ ). Re-writing the Amati relation (Amati et al. 2002) in terms of the isotropic energy, $E_{\text {iso }}$, and peak energy, $E_{\text {peak }}$, i.e. $E_{\text {peak }}=$ $95\left(E_{\text {iso }} / 10^{52} \mathrm{erg}\right)^{0.52}$ (Friedman \& Bloom 2005), we derive a rest-frame peak energy of $E_{\text {peak }}>306 \mathrm{keV}$. The total energy radiated in gamma-rays can be estimated from the Ghirlanda relation (Ghirlanda et al. 2004) which for GRB 051117A gives $E_{\gamma}=7.5 \times 10^{49} \mathrm{erg}$, which implies a beaming fraction $f_{\mathrm{b}}=0.01$. Both $E_{\text {iso }}$ and $E_{\gamma}$ place GRB 051117A amongst the low-end of the $E_{\text {iso }}$ and $E_{\gamma}$ distributions given in Frail et al. (2001).

The predicted jet opening angle and break-timescale for this source are $0.14^{\mathrm{c}}$ and 18.4 days respectively (assumes $\eta=0.2$, and $n=0.1 \mathrm{~cm}^{-3}$ ). This jet-break timescale is far later than the break in the late-time XRT light-curve and is only just covered by the late time data.

\section{Summary and conclusions}

GRB 051117A is one of the brightest GRBs (in terms of detected counts) observed through the Narrow Field X-ray Telescope at early times. The unprecedented $\mathrm{S} / \mathrm{N}$ of the early X-ray data has revealed complex temporal behaviour, both in the light-curve 
and the spectrum. The lightcurve displays multiple episodic flaring, indicative of a stochastic process. These flaring episodes result in an abrupt hardening of the X-ray spectrum which then slowly softens as the flares decline in intensity. Consequently, we find a significant correlation between source intensity and those parameters which characterise the properties of the spectrum, e.g. photon index $\Gamma$, hardness ratio $H R$ and break energy $E_{\text {break }}$. We find no evidence for variation in the hydrogen column in any of our data. Since spectral evolution is a characteristic common to many previously observed GRBs (particularly in the prompt emission), we suggest that the simplest explanation for the observed spectro-temporal variations is a model in which the break energy correlates with source intensity. That is, the breakenergy moves to higher energies at the onset of a flare before declining more slowly as the flare fades.

Finally, given the superb $\mathrm{S} / \mathrm{N}$ of our data we have searched for the presence of emission-line features in the early X-ray spectrum. We can rule out the presence of lines with $E W<$ $15 \mathrm{eV}$, assuming an intrinsic line width of $100 \mathrm{eV}$, at greater than $3 \sigma$ at the peak of the effective area.

Acknowledgements. We would like to thank the anonymous referee for very helpful comments which have helped to improve the work presented here. This work is supported at the University of Leicester by the Particle Physics and Astronomy Research Council (PPARC), at Penn State by NASA contract NAS500136, and in Italy by funding from ASI on contract number I/R/039/04.

\section{References}

Abbey, A. F., Carpenter, J., Read, A., et al. 2005, Proceedings of The X-ray Universe Conference, El Escorial, Spain, 2005, ESA SP-604, Vol. 1, 943 Amati, L., Frontera, F., Tavani, M., et al. 2002, A\&A, 390, 81

Band, D., Matteson, J., Schaefer, B., et al. 1993, ApJ, 413, 281

Band, D., Barthelmy, S., Beardmore A., et al. 2005, GCN, 4280

Barthelmy, S. D. 2004, Proc. SPIE, 5165, 175

Barthelmy, S. D., Barbier, L. M., Cummings, J. R., et al. 2005a, Sp. Sc. Rev., 120,143

Barthelmy, S. D., Chincarini, G., Burrows, D. N., et al. 2005b, Nature, 438, 994 Barthelmy, S. D., Canizzo, J. K., Gehrels, N., et al. 2005c, ApJ, 635, L133

Bhat, P. N., Fishman, G. J., Meegan, C. A., et al. 1994, ApJ, 426, 604

Burrows, D. N., Hill, J. E., Nousek, J. A., et al. 2004, SPIE, 5165, 201

Burrows, D. N., Hill, J. E., Nousek, J. A., et al. 2005a, Sp. Sc. Rev., 120, 165

Burrows, D. N., Romano, P., Godet, O., et al. 2005b, Proceedings of The X-ray

Universe Conference, El Escorial, Spain, 2005, ESA SP-604, Vol. 1, 877

Butler, N. R. 2007, ApJ, 656, 1001

Butler, N. R., Marshall, H. L., Ricker, G. R., et al. 2003, ApJ, 597, 1010

Campana, S., Romano, P., Covino, S., Lazzati, D., et al. 2006a, A\&A, 449, 61

Campana, S., Mangano, V., Blustin, A. J., et al. 2006b, Nature, 442, 1008

Crider, A., Liang, E. P., Smith, I. A., et al. 1997, ApJ, 479, L39

Cusumano, G., Mangano, V., Chincarini, G., et al. 2006, Nature, 440, 164

Cummings, J., Angelini, L., Barthelmy, S., et al. 2005, GCN, 3910

De Luca, A., Melandri, A., Caraveo, P. A., et al. 2005, A\&A, 440, 85

Dermer, C. 2004, ApJ, 614, 284

Drenkhahn, G., \& Spruit, H. C. 2002, A\&A, 391, 1141
Falcone, A. D., Burrows, D. N., Lazzati, D., et al. 2006, ApJ, 641, 1010 Fan, Y. Z., \& Wei, D. M. 2005, MNRAS, 364, L42

Ford, L. A., Band, D. L., Matteson, J. L., et al. 1995, ApJ, 439, 307

Friedman, A. S., \& Bloom, J. S. 2005, ApJ, 627, 1

Frail, D. A., Kulkarni, S. R., Sari, R., et al. 2001, ApJ, 562, L55

Fruchter, A. S., Thorsett, S. E., Metzger, M. R., et al. 1999, ApJ, 519, L13

Galama, T. J., \& Wijers, R. A. M. J. 2001, ApJ, 549, L209

Gehrels, N., Chincarini, G., Giommi, P., et al. 2004, ApJ, 611, 1005

Gehrels, N., Sarazin, C. L., O’Brien, P. T., et al. 2005, Nature, 437, 851

Gendre, B., Corsi, A., \& Piro, L. 2006, A\&A, 455, 803

Ghirlanda, G., Ghisellini, G., \& Lazzati, D. 2004, ApJ, 616, 331

Goad, M. R., Osborne, J. P., Beardmore, A. P., Godet, O., \& Page, K. 2006a, in Gamma-Ray Bursts in the Swift Era, ed. S. S. Holt, N. Gehrels, \& J. A. Nousek, American Institute of Physics

Goad, M. R., Tagliaferri, G., Page, K. L., et al. 2006b, A\&A, 449, 89

Goad, M. R., Page, K., Burrows, D., Hurley, K., \& Chester, M. 2005, GCN, 4287

Gao, W. H., \& Wei, D. M. 2005, Chin. J. Astron. Astrophys., 5, 6, 571

Guetta, D., D'Elia, V., Fiore, F., et al. 2006, in Il Nuovo Cimeto

Hurkett, C., et al. 2007, MNRAS, in press

King, A., O'Brien, P. T., Goad, M. R., et al. 2005, ApJ, 630, L113

Kobayashi, S., Piran, T., \& Sari, R. 1997, ApJ, 490, 92

Krimm, H. A., Barthelmy, S. D., Chester, M. M., et al. 2007, GCN, 6005

Kumar, P., \& Panaitescu, A. 2000, ApJ, 541, L51

Kumar, P., \& Narayan, R. 2003, ApJ, 584, 895

Kumar, P., McMahon, E., Panaitescu, A., et al. 2007, MNRAS, 376, L57

Holland, S. T. 2005, GCN, 4301

Holland, S. T., Barthelmy, S., Burrows, D. N., et al. 2006, GCN, 4570

Lamb, D., Donaghy, T. Q., \& Graziani, C. 2005, ApJ, 620, 355

Lazzati, D., \& Perna, R. 2002, MNRAS, 330, 383

Lazzati, D., \& Perna, R. 2007, MNRAS, 375, L46

Lazzati, D., Ramirez-Ruiz, E., \& Rees, M. J. 2002, ApJ, 572, L57

Lehto, H. J. 1989, in Symposium on Two Topics in X-ray Astronomy, Bolgna, Italy, Sep. 12-13 1989 (ESA SP-296 NOV 1989)

Mészáros, P., \& Rees, M. J. 1997, ApJ, 476, 232

Moretti, A., Perri, M., Capalbi, M., et al. 2006, A\&A, 448, L9

Nakar, E., \& Granot, J. 2006 [arXiv: astro-ph/0606011]

Nousek, J. A., Kouveliotou, C., Grupe, D., et al. 2006, 642, 389

Pei, Y. C. 1992, ApJ, 395, 130

Page, K. L., Barthelmy, S. D., Beardmore, A. P., et al. 2006, GCN, 5823

Piro, L., Costa, E., Feroci, M., et al. 1999, ApJ, 514, L73

Piro, L., Garmire, G., Garcia, M., et al. 2000, Science, 290, 955

Prochaska, J. X., Bloom, J. S., Chen, H.-W., et al. 2004, ApJ, 611, 200

Rees, M., \& Mészáros, P. 1994, ApJ, 430, L93

Rees, M., \& Mészáros, P. 2000, ApJ, 545, L73

Rees, M. J., \& Mészáros, P. 2005, ApJ, 628, 847

Reeves, J. N., Watson, D., Osborne, J. P., et al. 2002, Nature, 416, 512

Ryde, F., \& Petrosian, V. 2002, ApJ, 579, 290

Roming, P. W. A., Kennedy, T. E., Mason, K. O., et al. 2005, SSR, 120, 95

Stratta, G., Fiore, F., Antonelli, L. A., Piro, L., \& De Pasquale, M. 2004, ApJ, 608,846

Tagliaferri, G., Goad, M., Chincarini, G., et al. 2005, Nature, 436, 985 [arXiv: astro-ph/0506355]

Watson, D., Reeves, J. N., Hjorth, J., et al. 2003, ApJ, 595, L29

Willingale, R., O’Brien, P. T., Osborne, J. P., Godet, O., Page, K. L., et al. 2007, $\mathrm{ApJ}$, in press

Woosley, S. E., \& Bloom, J. S. 2006, ARA\&A, 44, 507

Yoshida, A., Namiki, M., Yonetoku, D., et al. 2001, ApJ, 557, L27

Zhang, B., \& Mészáros, P. 2004, IJMP, 19, 2385 